\section{Building a better battery}

By Don Monroe

$\mathrm{R}$ echargeable lithium-ion (Li-ion) batteries are ubiquitous. They power numerous portable electronic devices, such as laptop computers and phones. They are also likely to be key in our lower-carbon future, already powering most electric vehicles and, in the future, potentially helping to store grid-scale electric power from renewable but intermittent sources, such as wind and solar.

This widespread adoption of the Liion battery builds upon decades of work by many people with a variety of backgrounds and expertise including chemistry, physics, materials, and manufacturing, that find a home in the Materials Research Society (MRS). MRS Bulletin has featured numerous articles and special issues focusing on Li-ion batteries.

In 2019, three researchers-M. Stanley Whittingham* of Binghamton University (part of The State University of New York system), John B. Goodenough of The University of Texas at Austin, and Akira Yoshino of the Asahi Kasei Corporation in Tokyo and Meijo University in Nagoya, Japan-were recognized with the Nobel Prize in Chemistry for their contributions to this technology. Specifically, the three each made important advances in the electrode materials that made these batteries practical.

During battery use, or discharge, positive ions are liberated from the negative electrode, or anode, and are subsequently

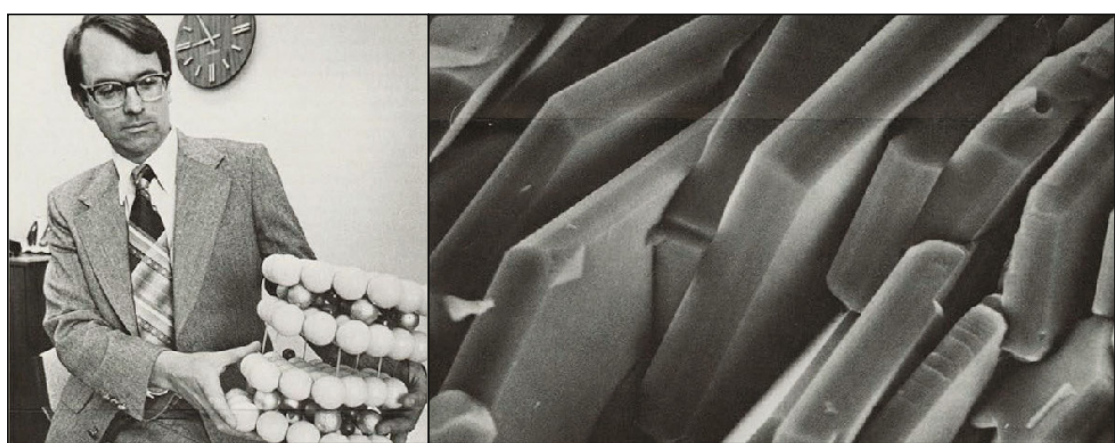

M. Stanley Whittingham at Exxon's research laboratories in 1979. Credit: ExxonMobil Corporation.
University, Whittingham had explored intercalation of other alkali metals into $\mathrm{TaS}_{2}$. His experiments at Exxon showed that an electrochemically driven version of this process could pull $\mathrm{Li}^{+}$ions in and out of the lighter material, $\mathrm{TiS}_{2}$. Whittingham combined his intercalated $\mathrm{TiS}_{2}$ cathode with a metallic Li anode to create a battery with a voltage of approximately $2.5 \mathrm{~V}$.

Researchers at Stanford and elsewhere had previously used intercalation to synthesize new materials, including superconductors, from layered chalcogenides. (In recent years, many of these same materials have been used to form exotic two-dimensional sheets of atoms, similar to graphene, by breaking the van der Waals attraction between their covalently bonded layers.) Intercalation has important advantages for ion storage, since the ions can repeatedly enter and leave without disrupting the layered structure, although the extra atoms also dilute the available charge-storage density. The overall structure must allow rapid transport of both ions and electrons and avoid any side reactions that may be facilitated by the large amount of electrical energy available.

The electrolyte must also resist reactions. The large voltage makes this a particular challenge for aqueous solvents, because water undergoes electrolysis at voltages above approximately $1.2 \mathrm{~V}$ and also reacts with metallic lithium. Whittingham's battery used an organic electrolyte, propylene carbonate, which had previously been shown to allow rapid $\mathrm{Li}^{+}$transport in the form of an added lithium salt.

Exxon patented Whittingham's battery, but it failed commercially, as did products with other cathode materials introduced during the same period. A major problem was the metallic anode. Although metallic lithium electrodes are used in non-rechargeable "primary" batteries, recharging involves regrowth of metallic lithium at the anode. During this process, the metal tends to form whiskers or dendrites that extend into the electrolyte. These can become electrically isolated from the electrode during subsequent discharge, reducing capacity. More importantly, during repeated charging cycles, the dendrites can grow all the way to the cathode, causing a catastrophic short circuit. This 
problem has still not been fully solved, so the original vision of the rechargeable "secondary" battery that takes advantage of the low ionization energy and low density of lithium metal remains unrealized.

In 1976, Goodenough had moved to the University of Oxford, after nearly a quarter century studying magnetic materials at the Massachusetts Institute of Technology Lincoln Laboratory. Extending Whittingham's results, he recognized that the layered structure of $\mathrm{CoO}_{2}$ might offer the same opportunities for $\mathrm{Li}^{+}$intercalation as the dichalcogenides, but that the more negative oxygen could provide an even more welcoming environment. (Like sulfur, selenium, and tellurium, oxygen is a Group-VI element, but is not often included with them as a chalcogenide.)

Goodenough found that he could make a battery delivering more than $4 \mathrm{~V}$ of potential relative to a metallic lithium anode. The $\mathrm{Li}_{x} \mathrm{CoO}_{2}$ cathode material is not perfect. It is a poor electrical conductor, so it must be interspersed with an electrically conducting material that carries the electrons close to the lithium ions. Cobalt makes the material relatively heavy, and it is not practical to cycle the composition below $x=1 / 2$, which halves the charge capacity. Lithium batteries have also been implicated in fires, including in Tesla cars, Boeing aircraft, hoverboards, and electronic devices, in part because oxygen released from the cathode can react with the polymer electrolyte. Therefore, researchers, including Goodenough, continued to explore alternatives, including spinels and olivines. Nonetheless, the $\mathrm{Li}_{x} \mathrm{CoO}_{2}$-intercalated cathode material remains a key enabler of the commercial success of Li-ion batteries.

Commercial adoption took time even after Whittingham and Goodenough's work, however, because of safety and reliability concerns about Li-metal anodes. Earlier researchers had suggested a "rocking chair" operation, in which the anode, as well as the cathode, incorporated $\mathrm{Li}^{+}$ions by intercalation. In particular, Samar Basu, Bell Labs in New Jersey, and Rachid Yazami, the Centre National de la Recherche Scientifique in Grenoble, France, showed that layers of graphite formed a suitable host for lithium ions, with only a small voltage penalty relative to lithium metal.
Unfortunately, graphite also admitted molecular components of the electrolyte that were also intercalated into the graphite, causing destructive exfoliation of the layers in graphite.

At Asahi Kasei Corporation in Japan in the mid1980s, Yoshino explored several other possible anode materials before finding that heat-treated petroleum coke could prevent exfoliation. Sometimes categorized as "soft carbon," this complex material includes both graphitic regions and morerigid carbon regions that stabilize the graphite during intercalation. In 1991, Sony, which saw possibilities for rechargeable batteries for its portable consumer-electronics products, introduced a Liion battery for a camcorder based on Yoshino's anode, in what is widely regarded as a watershed moment for the technology. Following this successful commercialization, other companies followed suit, and in five years, these batteries were outselling the competing nickel cadmium rechargeable batteries.

The technology has continued to improve. Although the charge capacity per $\mathrm{kg}$ of a graphite electrode is an order of magnitude lower than that of pure lithium metal, the energy-storage capacity per $\mathrm{kg}$ of Li-ion batteries immediately exceeded that of the existing lead-acid and nickel cadmium batteries and is now an order of magnitude higher.

Subsequent generations of batteries also returned closer to a graphite anode. Reliability was improved through the use of ethylene-carbonate-based electrolytes, which fortuitously form a self-limiting solid-electrolyte interphase region at the electrode surface. This layer acts as a thin, solid electrolyte that permits transit of small lithium ions while blocking the components of the electrolyte that lead to exfoliation.
Advances in Li-ion batteries, beginning with the introduction of intercalated electrodes, have profoundly changed our technological world. Researchers continue to explore both incremental and revolutionary improvements in the technology. These include tweaks to the electrode and electrolyte processing and composition to improve reliability and lifetime, as well as entirely new cathode materials. The incorporation of regulatory electronics into the battery package also provides improved safety under extreme charging conditions or after physical damage, compensating for some of the materials-related weaknesses. Indeed, with a proper understanding of these complex materials and clever design of the devices, we may one day realize, in a practical form, the original dream of a Li-metal rechargeable battery.

*Member of the MRS Bulletin Editorial Board. 